\title{
SHOCK GEOMETRY AND SPECTRAL BREAKS IN LARGE SEP EVENTS
}

\author{
G. Li ${ }^{1}$, G. P. Zank ${ }^{1}$, Olga Verkhoglyadova ${ }^{2}$, R. A. Mewaldt ${ }^{3}$, C. M. S. Cohen ${ }^{3}$, G. M. Mason Mnd An M. I. Desai $^{5}$ \\ ${ }^{1}$ Department of Physics and Center for Space Plasma and Aeronomic Research, University of Alabama in Huntsville, Huntsville, AL 35899, USA \\ ${ }^{2}$ Center for Space Plasma and Aeronomic Research, University of Alabama in Huntsville, Huntsville, AL 35805, USA \\ ${ }^{3}$ California Institute of Technology, Pasadena, CA 91125, USA \\ 4 John Hopkins University and Applied Physics Lab, Laurel, MD 20723, USA \\ 5 South West Research Institute, San Antonio, TX 78249, USA \\ Received 2009 February 1; accepted 2009 July 9; published 2009 August 18
}

\begin{abstract}
Solar energetic particle (SEP) events are traditionally classified as "impulsive" or "gradual." It is now widely accepted that in gradual SEP events, particles are accelerated at coronal mass ejection-driven (CME-driven) shocks. In many of these large SEP events, particle spectra exhibit double power law or exponential rollover features, with the break energy or rollover energy ordered as $(Q / A)^{\alpha}$, with $Q$ being the ion charge in $e$ and $A$ the ion mass in units of proton mass $m_{p}$. This $Q / A$ dependence of the spectral breaks provides an opportunity to study the underlying acceleration mechanism. In this paper, we examine how the $Q / A$ dependence may depend on shock geometry. Using the nonlinear guiding center theory, we show that $\alpha \sim 1 / 5$ for a quasi-perpendicular shock. Such a weak $Q / A$ dependence is in contrast to the quasi-parallel shock case where $\alpha$ can reach 2 . This difference in $\alpha$ reflects the difference of the underlying parallel and perpendicular diffusion coefficients $\kappa_{\|}$and $\kappa_{\perp}$. We also examine the $Q / A$ dependence of the break energy for the most general oblique shock case. Our analysis offers a possible way to remotely examine the geometry of a CME-driven shock when it is close to the Sun, where the acceleration of particle to high energies occurs.
\end{abstract}

Key words: acceleration of particles - Sun: coronal mass ejections (CMEs)

\section{INTRODUCTION}

Diffusive shock acceleration, also known as first-order Fermi acceleration, was first proposed some 30 years ago (Axford et al. 1977; Krymsky 1977; Bell 1978a, 1978b; Blandford \& Ostriker 1978). Being a remarkably simple and elegant theory, it predicts a power-law spectrum of galactic cosmic rays that agrees well with observations. Indeed, it has been suggested that supernova blast shock waves are responsible for the acceleration of cosmic rays to energies up to $\sim 10^{15} \mathrm{eV}_{\text {nucleon }}{ }^{-1}$ (Ip \& Axford 1992). On a much smaller scale, diffusive shock acceleration occurs in gradual solar energetic particle (SEP) events, where particles are accelerated at a propagating CME-driven shock by repeatedly traversing the shock front. The spectra of energetic particles observed in these events often show power laws with a break or rollover at a few to a few tens of $\mathrm{MeV}$ nucleon $^{-1}$ (Mazur et al. 1992; Tylka et al. 2000; Cohen et al. 2005; Mewaldt et al. 2005a; Tylka et al. 2005). An empirical fit using an exponential rollover of the form

$$
d J / d E \approx E^{-\gamma} \exp \left(-E / E_{0}\right)
$$

where $d J / d E$ is the particle differential number intensity current, and $E_{0}$ is the break energy that was suggested by Ellsion \& Ramaty (1985). However, as shown by Cohen et al. (2005) and Mewaldt et al. (2005b), a double power law offers a better fit to observations for many events. In particular, Tylka et al. (2006) found that the following form, used first in gamma ray burst studies (Band et al. 1993),

$$
\begin{gathered}
d J / d E=C E^{-\alpha} \exp \left(-E / E_{0}\right) \\
\quad \text { for } E \leqslant(\beta-\alpha) E_{0} \\
d J / d E=C E^{-\beta}\left[(\beta-\alpha) E_{0}\right]^{\beta-\alpha} \exp (\alpha-\beta) \\
\text { for } E \geqslant(\beta-\alpha) E_{0},
\end{gathered}
$$

often describes the spectra better. Indeed, in a study of the series of Halloween events, Cohen et al. (2005) and Mewaldt et al. (2005b) found that in 10 of 15 cases the Band function yielded a better fit. Equation (3) is a double power law joined by an exponential. Neither this double power law nor the exponential rollover is predicted by the original steady-state diffusive shock acceleration theory, which further assumed a simple one-dimensional geometry and a planar shock. Earlier attempts to explain an exponential rollover of energetic particle spectra in a shock environment can be found in Forman et al. (1981), Forman \& Webb (1985), Lee (1983), Ellsion \& Ramaty (1985), and Channok et al. (2005), where the effects of adiabatic deceleration, finite acceleration timescale, and finite shock size are considered. However, none of these studies examined the $Q / A$ dependence of the spectra. This is partly because earlier observations did not have collecting power needed to measure heavy-ion spectra accurately up to high energies.

The first systematic analysis of spectral rollovers that related them to $Q / A$ and discussed how they might probe conditions at the shock was done by Tylka et al. (2000). In Tylka et al. (2000), the time evolution of energetic particle spectrum in two SEP events (1998 April 20 and 1998 August 25) was examined, and it was found that the e-folding energies show some decreases as the event goes on. Later Tylka et al. (2005) first made the explicit connection between shock geometry and high-energy SEP spectral variability; this work was further extended by Tylka $\&$ Lee (2006), who modeled the connection between shockgeometry and spectral variability, although no quantitative $Q / A$ dependence of spectral rollovers was pursued. These works by Tylka and coworkers have laid the ground for the present work.

In a theoretical effort to explain the observed double power law of particle spectra in certain events, Li et al. (2005a) put forth a mechanism by considering the effect of a loss term in the transport equation. The loss term is introduced to reflect the leakage of accelerated particles from the shocked upstream 
region. They found that a broken power law can be obtained if the diffusion coefficient has a "step-like" behavior as a function of energy. This exercise showed that a break in the particle energy spectrum can be related to the rigidity dependence of the particle diffusion coefficient. If for different species this "step-like" behavior of the diffusion coefficient occurs at different energies, then a $Q / A$ dependence of the break energy comes out naturally. Indeed, in another work, by fitting heavy ion spectra to a double power law or an exponential rollover, Cohen et al. (2003) found that the break energies in several events can be ordered by $(Q / A)^{\delta}$, with $\delta$ varying from one event to another. In the Cohen et al. (2003) study, the breaks occur at the same value of the diffusion coefficient $\kappa$. By equating $\kappa$ for different heavy ions, Cohen et al. (2003) were able to relate the break energy to the spectral index of the turbulence power spectra. This relationship is important because it offers us a unique way to remotely probe the turbulence power spectrum at the acceleration site by using energetic particle spectra. Extending the work of Cohen et al. (2003, 2005), Mewaldt et al. (2005b) have examined the $Q / A$ dependence of particle spectra for a series of large SEP events that occurred between 2003 October 28 and 2003 November 4. They found that heavy ion spectra in all these events show very good $(Q / A)^{\delta}$ ordering, with $\delta$ varying from 1 to 2 . These results agree with earlier findings by Tylka et al. (2000), who showed that in one event the exponent was time-independent around unity and in the other event the exponent evolved from $\sim 2.5$ to $\sim 1.0$. The variability in $\delta$ suggests that the underlying acceleration processes in different events are different. In a related work, Mason et al. (2006) discussed how the $Q / A$ dependence of the diffusion coefficient $\kappa$ can be used to organize the observed time intensity profile for different ions in SEP events. They found that in many SEP events, the time intensities of different ions $(Q / A)$ at different energy per nucleon track each other remarkably well as if these ions undergo the same transport process. If this process is governed by diffusion, which is solely controlled by $\kappa$, then an ordering of $Q / A$ will emerge if $\kappa$ depends on rigidity.

Clearly, these earlier studies reveal that $Q / A$ ordering of certain observations in SEP events provides an important venue to understand the underlying particle acceleration and transport. They have motivated this present work. In this paper, we examine how the $Q / A$ dependence of the breaks in energetic particle spectra may depend on shock geometry. We consider both quasi-parallel and quasi-perpendicular shocks as well as the most general oblique shock case. We discuss several physical considerations that lead to a spectral break. We show that the $Q / A$ dependence of the break energy is through the $Q / A$ dependence of the diffusion coefficient $\kappa$. In describing the perpendicular diffusion coefficient $\kappa_{\perp}$, we use the nonlinear guiding center (NLGC) theory (Matthaeus et al. 2003; Zank et al. 2004). We show that in this theory, a $(Q / A)^{1 / 5}$ dependence for the break energy arises. This is in contrast to the quasi-parallel case, where $(Q / A)^{2}$ can be found. In the oblique shock case, the ordering of the break energy with respect to $Q / A$ depends on the relative size of $\kappa_{\perp}$ and $\kappa_{\|}$, as well as the angle $\theta_{B N}$.

\section{WHAT CONTROLS THE BREAKS IN ENERGETIC PARTICLE SPECTRA}

In an earlier work Tylka et al. (2000) studied the time evolution of energetic particle spectra in two SEP events (1998 April 20 and 2000 August 25). They found that the e-folding energies show some decreases as the event goes on. Several things may contribute to this: in the early part of the event, velocity dispersion will lead to flatter spectra ${ }^{6}$; later on, the highenergy particles decay away before the low-energy ones do, and this will continue the lowering of the break energy. Clearly, to understand this evolution, transport is a factor. The time dependences of the e-folding energy in the Tylka et al. (2000) paper are pretty small after the initial portion, and the relative spacing of $\mathrm{He}$, versus $\mathrm{O}$ versus $\mathrm{Fe}$ shows little variation-that is, they all tend to move together. Finally, we note that the two events studied in Tylka et al. (2000) were not "well connected" events; therefore, the effects of the "magnetic connection" may have been significant.

It is beyond the scope of this paper to employ a detailed transport calculation to address the issue of the time evolution of energetic particle spectrum. We therefore limit ourselves here to event-integrated spectra only. Event-integrated spectra ignore the effects of transport. However, since a CME-driven shock weakens as it propagates out, at its front the maximum achievable particle energy decreases with time (for both the parallel and perpendicular shocks); therefore, it is conceivable that the effect of energetic particles from later acceleration on the energy break is minimal. Note that the value of the shocknormal angle is also expected to change as the shock moves outward (see, for example, the simulations by Manchester et al. 2005).

As shown in Li et al. (2005b), the spectral shape for heavy ions is determined by the "equal resonance" condition, i.e., the break energy is decided by the smallest wave number $k_{0}$ that resonates with various heavy ions. The resonance condition is

$$
k_{0}=\frac{Q}{A} \frac{e B}{\mu \tilde{p} c},
$$

where $Q$ is the ion's charge in the unit of $e, A$ is the ion's mass number, $\tilde{p}$ is the momentum per nucleon, $\mu$ is the particle's pitch angle cosine, and $c$ is the speed of light.) In Li et al. (2005b), following Gordon et al. (1999) and Lee (1983), $\mu=1$ was used in Equation (4). This assumption is based on the fact that for a given particle momentum $\tilde{p}$, the power of the resonant turbulence is the maximum at $\mu=1$, provided that the power spectrum $I(k)$, as a function of $k$, is decreasing with $k$. Such an assumption is not needed here, as the $Q / A$ dependence of $\tilde{p}$ does not require any assumption about $\mu$.

This $k_{0}$ is self-generated by protons streaming upstream of the shock. Because the wave power at $k<k_{0}$ in Li et al. (2005b) corresponds to the ambient solar wind turbulence and at $k>k_{0}$ corresponds to the self-generated Alfvén waves/turbulence, the wave power spectrum has a discontinuity at $k_{0}$. This sudden change of the wave power at $k_{0}$ causes a break in the particle energy spectrum with a $(Q / A)^{2}$ dependence, as shown by the simulations of Li et al. (2005b). Of course, this discontinuity of $I(k)$ at $k_{0}$ in Li et al. (2005b) is only an approximation. More rigorous, self-consistent treatments of the wave growth show a sharp, but not discontinuous rise in the wave power at $k_{0}$ (see, for example, Figure 2 of $\mathrm{Ng} \&$ Reames 2008).

The above "equal resonance" condition for the spectral break can be also understood from the following considerations which offer additional physical insights. We refer to these as the "equal diffusion coefficient" condition; the "equal acceleration time" condition; and finally the "equal gyroradius" condition. Unlike

\footnotetext{
6 Note that Tylka et al. (2000) only used particles above $2.4 \mathrm{MeV}$ nucleon $^{-1}$, therefore the nominal travel time (for $1.2 \mathrm{AU}$ ) is about $2 \mathrm{hr}$. So the

velocity-dispersion effects in the (Tylka et al. 2000) spectra are expected to be small.
} 
the "equal resonance" condition, these latter three conditions apply to both quasi-parallel and quasi-perpendicular shocks, and the more general oblique shock case. As discussed above, Cohen et al. (2003, 2005) employed the "equal diffusion coefficient" condition. The idea is that the escape of particles from a shock is governed by the particle diffusion coefficient $\kappa$. The break energies of various heavy ions occur at the same $\kappa$ value. Thus, if $\kappa$ has a certain $Q / A$ dependence, this will translate to the break energy $E_{0}$ that depends on $Q / A$.

We now consider the "equal acceleration time" condition. Here the break energy $E_{0}$ is decided by requiring various heavy ions to have the same acceleration time. Since the acceleration time is given by (see Equation (3.46) of Drury 1983)

$$
\Delta t=\frac{3 s}{s-1} \frac{\kappa_{\mathrm{up}}(p)+s \kappa_{\mathrm{dn}}(p)}{u_{\mathrm{sh}}^{2}} \frac{\Delta p}{p},
$$

where $s$ is the compression ratio of the shock, $u_{\mathrm{sh}}$ is the shock speed as seen in the upstream frame, $\kappa_{\text {up }}$ and $\kappa_{\mathrm{dn}}$ are particle diffusion coefficients in the upstream and downstream regions of the shock, given the same acceleration time for different heavy ions, one can use Equation (5) to obtain the maximum momentum $p_{m}$ through

$$
t=\int_{p_{0}}^{p_{m}} \frac{3 s}{s-1} \frac{\kappa}{u_{\mathrm{sh}}^{2}} \frac{1}{p} d p,
$$

where

$$
\kappa=\kappa_{\mathrm{up}}(p)+s \kappa_{\mathrm{dn}}(p) .
$$

At a quasi-parallel shock, $\kappa=\kappa_{\|} \cos ^{2} \theta_{B N}+\kappa_{\perp} \sin ^{2} \theta_{B N}$ is approximately $\kappa_{\|}$and the upstream (downstream) $\kappa_{\text {up }}\left(\kappa_{\mathrm{dn}}\right)$ is related to the self-generated wave intensity $I(k)$ (Lee 1983, 2005), which increases significantly (see e.g., McKenzie \& Westphal 1969) from upstream to downstream of the shock, leading to $\kappa_{\text {up }} \gg \kappa_{\text {dn }}$ and thus $\kappa \sim \kappa_{\text {up }}$; at a quasi-perpendicular shock, the self-generated waves are suppressed by $\cos \left(\theta_{B N}\right)$, thus the contribution to the total diffusion coefficient from $\kappa_{\|}$can be smaller than the contribution from the perpendicular diffusion coefficients. In this work, we adopt the NLGC theory (Matthaeus et al. 2003; Zank et al. 2004) for the perpendicular diffusion coefficient $\kappa_{\perp}$. In the NLGC theory and its recent variants (Salchi 2004, 2006), the value of $\kappa_{\perp}$ is decided by the two-dimensional component of the turbulence (a two-dimensional turbulence has a wave vector $k$ perpendicular to the background $B_{0}$ ), which is independent of the slab component (where the wave vector $k$ is parallel to $B_{0}$ ). While Alfvén waves parallel to $B_{0}$ (thus of slab geometry) are subject to amplification due to streaming protons, the two-dimensional component of the turbulence is not. Over a broad range of energy, the diffusion coefficient $\kappa_{\perp}$ at a quasiperpendicular shock can be related to the particle parallel mean free path through (Zank et al. 2004)

$$
\kappa_{\perp}=\frac{1}{3} v \lambda_{\perp}=\frac{C}{3} v l_{2 \mathrm{D}}^{2 / 3}\left(\lambda_{\|}\right)^{1 / 3}
$$

where $C$ is a constant, $l_{2 \mathrm{D}}$ is the bend-over scale of the twodimensional turbulence, and $\lambda_{\|}$is the particle's parallel mean free path. At a perpendicular shock, $\cos \theta_{B N}=0$, therefore $\lambda_{\|}$ in Equation (8) is just $\lambda_{\|}^{0}$, the parallel mean free path in the quiet solar wind. In this case, assuming $l_{2 \mathrm{D}}$ is similar in the upstream and downstream, then $\kappa_{\text {up }} \sim \kappa_{\text {dn }}$ and we have $\kappa \sim(1+s) \kappa_{\text {up }}$.

From Equation (6) it is clear that if $\kappa$ has a $Q / A$ dependence then requiring $t$ to be the same for different ions will lead to a $Q / A$ dependence for the break energy.
Finally, there is the "equal gyroradius" condition. If the MHD turbulence near a CME-driven shock is so strong such that the turbulent magnetic field $\delta B$ is comparable to the background magnetic field $\sim B_{0}$, then referring to a shock as being quasiparallel or quasi-perpendicular becomes meaningless. In this case, the intrinsic length scale for a charged particle is given by the particle's gyroradius,

$$
r_{g}=\frac{A}{Q} \frac{\tilde{p} c}{e B},
$$

where $p$ is the particle's momentum, $\tilde{p}$ is the particle's momentum per nucleon, $Q e$ is the particle's charge and $B=B_{0}+\delta B$ is the total magnetic field. Clearly, charged particles at the same location and having the same curvature $q=1 / r_{g}$ will have the same trajectory. If the acceleration and escape process are determined by the curvature, then the $Q / A$ dependence of the break energy will be controlled by $r_{g}$. Requiring $r_{g}$ to be the same for different heavy ions, we find $E_{0}$, the break energy (per nucleon),

$$
E_{0}=\tilde{p}^{2} / 2 m_{p} \sim(Q / A)^{2},
$$

where $\tilde{p}$ is momentum per nucleon. The $Q / A$ dependence in Equation (10) is the same as in the "equal resonance" condition.

In the following, we consider a generic form of $\kappa$ as $\kappa=$ $\kappa_{0}\left(v / v_{0}\right)^{\gamma}(A / Q)^{\epsilon}$. For example, at a parallel shock where selfenhanced wave power exists, we have (Lee 2005)

$$
\kappa_{\|} \sim(A / Q)^{2} \frac{v^{3}}{I(k=\Omega / v)},
$$

with $I(k) \sim k^{\beta}$, then

$$
\kappa_{\|}=\kappa_{\|, 0}\left(v / v_{0}\right)^{\beta+3}(A / Q)^{\beta+2} .
$$

The Bohm approximation is recovered when $\beta=-1$. At a (strictly) perpendicular shock, using Equation (8) with $\lambda_{\|} \sim$ $\left(r_{g}\right)^{1 / 3}$, one has

$$
\kappa_{\perp}=\kappa_{\perp, 0}\left(v / v_{0}\right)^{10 / 9}(A / Q)^{1 / 9} .
$$

In general, we can write $\kappa$ as the follows:

$$
\kappa=\kappa_{0}\left(v / v_{0}\right)^{\gamma}(A / Q)^{\epsilon},
$$

where $\gamma=2$ and $\epsilon=1$ corresponds to the Bohm approximation; $\gamma=\beta+3, \epsilon=\beta+2$ corresponds to the case of a parallel shock, $\gamma=10 / 9$ and $\epsilon=1 / 9$ corresponds to the case of a perpendicular shock.

From Equation (3), we find that the "equal diffusion coefficient" condition yields

$$
(A / Q)^{\epsilon}\left(\tilde{p}_{m}\right)^{\gamma}=\text { constant. }
$$

Assuming equal acceleration time and using Equation (3) in Equation (6), we get

$$
t_{\mathrm{acc}}=\frac{3 s}{s-1} \frac{\kappa_{0}}{u_{\mathrm{sh}}^{2}}\left(\frac{A}{Q}\right)^{\epsilon} \frac{1}{\gamma}\left(v_{m}^{\gamma}-v_{0}^{\gamma}\right),
$$

where $v_{0}$ is the injection speed of the particle and $v_{m}$ is the maximum speed. In obtaining Equation (16), we assume the particle speed $v$ is much smaller than the speed of light and work within the non-relativistic regime, so that $v=\tilde{p} / m_{n}$, 
where $m_{n}=938 \mathrm{MeV} \mathrm{c}^{-2}$ is the proton mass. If $v_{m} \gg v_{0}$, Equation (16) leads to

$$
(A / Q)^{\epsilon}\left(\tilde{p}_{m}\right)^{\gamma}=\text { constant. }
$$

Therefore, the "equal acceleration time" condition and the "equal diffusion coefficient" condition yield the same result. One can also show that the "equal resonance" condition is a special case for the "equal acceleration time" condition (see below). In the following, we therefore focus our study on the "equal diffusion coefficient" condition. From Equation (17), we find that the $Q / A$ dependence of the break energy $E_{0}$ (energy per nucleon) is

$$
E_{0}=\tilde{p}_{m}^{2} \sim\left(\frac{Q}{A}\right)^{2 \epsilon / \gamma}
$$

For the Bohm approximation, $\gamma=2$ and $\epsilon=1$, we find $E_{0} \sim(Q / A)$. Such dependence has been reported, for example, in Tylka et al. (2005). At a parallel shock with $I(k) \sim k^{\beta}$, we have

$$
E_{0} \sim(Q / A)^{2(2+\beta) /(3+\beta)}
$$

When $\beta=-1$, the Bohm approximation is recovered. A $\beta$ value of -1 is harder than the quiet solar wind value, which is close to $-5 / 3$ or -1.5 . However, when upstream waves are present, the spectrum can become harder and $\beta=-1$ is possible. If we take $\beta=-1.5$, we have $E_{0} \sim(Q / A)^{2 / 3}$; take $\beta=0$, we have $E_{0} \sim(Q / A)^{4 / 3}$. Observationally, 11 SEP events with gamma values ranging from 0.03 to 1.7 have been reported by Mewaldt et al. (2007) and several examples are discussed in Section 3. One can also invoke a positive $\beta$ (Cohen et al. 2003). Such an $I(k)$ has been suggested to exist in the early stage of proton-excited wave spectrum in some SEP events by $\mathrm{Ng}$ et al. (2003). Moreover, if one takes $\beta= \pm \infty$, which corresponds to a discontinuity of $I(k)$ (as modeled in Li et al. 2003 and Rice et al. 2003), then one finds $E_{0} \sim(Q / A)^{2}$, recovering the "equal resonance" condition. Thus, the "equal resonance " condition is a special case $(\beta=\infty)$ for the "equal acceleration time" condition or the "equal diffusion coefficient" condition. This is hardly a surprise if we note that in the "equal resonance" condition, the wave power $I(k)$ at $k=k_{0}$ (therefore $\kappa(p)$ at $p_{0}$ ) is discontinuous, since at $k<k_{0}$ it is taken to be the ambient solar wind turbulence wave power, and at $k>k_{0}$ it is taken from the self-generated Alfvén wave. This sudden change in $\kappa$ can be regarded as $\beta= \pm \infty$ for a $I(k) \sim k^{-\beta}$.

Perhaps the most interesting case is for a perpendicular shock, where $\gamma=10 / 9$ and $\epsilon=1 / 9$. In this case, one finds $E_{0} \sim(Q / A)^{1 / 5}$. This represents a very weak dependence on $Q / A$, one that is very different from a quasi-parallel shock case where a $Q / A$ to $(Q / A)^{2}$ dependence is often found. As such, it provides a feasible way of identifying perpendicular shock acceleration in gradual SEP events. An example of an SEP event with gamma $\sim 0.22$ is shown in Section 3 .

\section{SPECTRAL BREAKS AT OBLIQUE SHOCKS}

The most general case of an interplanetary shock is an oblique shock. For practical purposes, in this work we refer to a shock as an oblique shock when the angle between the magnetic field of the upstream plasma and the shock normal, i.e., $\theta_{B N}$, is between $20^{\circ}$ and $70^{\circ}$
At an oblique shock, the total diffusion coefficient, $\kappa$ can be written as

$$
\begin{aligned}
\kappa= & \kappa_{\|, 0}\left(v / v_{0}\right)^{\gamma_{\|}}\left(\frac{A}{Q}\right)^{\epsilon_{\|}} \cos ^{2}\left(\theta_{B N}\right) \\
& +\kappa_{\perp, 0}\left(v / v_{0}\right)^{\gamma_{\perp}}\left(\frac{A}{Q}\right)^{\epsilon_{\perp}} \sin ^{2}\left(\theta_{B N}\right),
\end{aligned}
$$

where we have used Equation (14) for $\kappa_{\|}$and $\kappa_{\perp}$ with $\kappa_{\|, 0}$ and $\kappa_{\perp, 0}$ the reference parallel and perpendicular diffusion coefficients at $v_{0}$. If we denote the ratio of $\kappa_{\perp, 0}$ to $\kappa_{\|, 0}$ as $r_{d}$,

$$
r_{d}=\frac{\kappa_{\perp, 0}}{\kappa_{\|, 0}}
$$

then Equation (20) becomes

$$
\begin{aligned}
\kappa= & \kappa_{\|, 0}\left[\left(v / v_{0}\right)^{\gamma_{\|}}\left(\frac{A}{Q}\right)^{\epsilon_{\|}} \cos ^{2}\left(\theta_{B N}\right)\right. \\
& \left.+r_{d}\left(v / v_{0}\right)^{\gamma_{\perp}}\left(\frac{A}{Q}\right)^{\epsilon_{\perp}} \sin ^{2}\left(\theta_{B N}\right)\right] .
\end{aligned}
$$

Envoking the "equal diffusion coefficient" condition again, one obtains

$$
\left(v / v_{0}\right)^{\gamma_{\|}}\left(\frac{A}{Q}\right)^{\epsilon_{\|}}+\xi\left(v / v_{0}\right)^{\gamma_{\perp}}\left(\frac{A}{Q}\right)^{\epsilon_{\perp}}=\text { constant. }
$$

In Equation (23), we have defined a new parameter $\xi$,

$$
\xi=r_{d} \tan ^{2}\left(\theta_{B N}\right)
$$

When $\gamma_{\|}, \epsilon_{\|}, \gamma_{\perp}$, and $\epsilon_{\perp}$ are given, the $Q / A$ dependence of the break energy is decided by $\xi$.

In obtaining Equation (23), we used $\kappa_{\perp}=\kappa_{\perp, 0}\left(v / v_{0}\right)^{\gamma_{\perp}}\left(\frac{A}{Q}\right)^{\epsilon_{\perp}}$. We now discuss what values of $\gamma_{\perp}$ and $\epsilon_{\perp}$ should be used at an oblique shock. Equation (23) can be derived by rewriting Equation (8) to

$$
\kappa_{\perp}=\kappa_{\perp, 0}\left(\frac{v}{v_{0}}\right)^{2 / 3}\left(\frac{\lambda_{\|}}{\lambda_{\|, 0}}\right)^{1 / 3},
$$

where $\lambda_{\|, 0}$ is the reference particle's parallel mean free path at $v=v_{0}$.

At a strict perpendicular shock as discussed in the last section, since there are no enhanced waves due to streaming protons, $\lambda^{\prime \prime}$ in Equation (25) is taken to be the quiet time particle mean free path $\lambda^{0}$, yielding the expression of $\kappa_{\perp}$ in Equation (14).

At an oblique shock, the total power in the direction parallel to $B_{0}$ can be written as $I(k)=I_{0}(k)+I_{e}(k)$, where $I_{0}(k)$ is the background solar wind turbulence power and $I_{e}(k)$ is the enhanced wave intensity generated at the shock front by streaming protons. Although suppressed by $\cos \left(\theta_{B N}\right), I_{e}(k)$ can be larger than $I_{0}(k)$. In this case, one may question the validity of the NLGC theory, and, if it holds, whether one should use $\lambda_{\|}^{e}$ due to the enhanced wave intensity to replace $\lambda_{\|}^{0}$ in determining the break energy. As we discuss below, the NLGC theory indeed can be applied to an oblique shock. However, the substitution of $\lambda_{\|}^{0}$ by $\lambda_{\|}^{e}$ is not necessarily a better approximation.

Consider first the validity of the NLGC theory at an oblique shock. In the original derivation of the NLGC theory or its recent variants (Matthaeus et al. 2003; Zank et al. 2004; Salchi 2004, 2006), a "slab+2D" turbulence geometry is assumed where the 


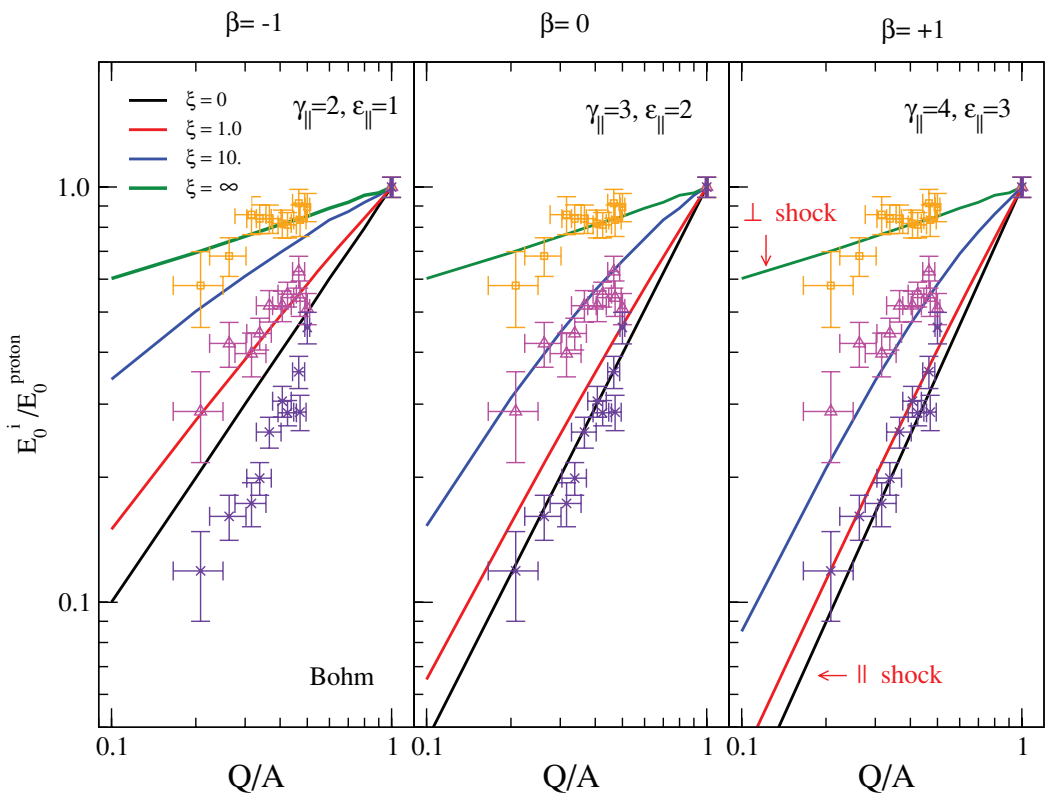

Figure 1. Break energy $E_{0}$ (normalized to the proton break energy $E_{0}$ ) as a function of $Q / A$ for an oblique shock. In all three panels, $\gamma_{\perp}=10 / 9$ and $\epsilon_{\perp}=1 / 9$ are assumed; from the left to the right the values of $\beta\left(I(k) \sim k^{\beta}\right)$ are $-1,0,+1$ respectively; the corresponding $\gamma_{\|\|}$and $\epsilon_{\|}$s are also shown. The black curve is for $\xi=0$, corresponding to a parallel shock; the green curve is for $\xi=\infty$, corresponding to a perpendicular shock; and the red and blue curves are for $\xi=1.0$ and $\xi=10$. Also plotted in the figure are the break energies for three events: 2001 April 2, 2002 November 9 and 2002 April 21 respectively. They are represented by the squares, triangles, and stars.

slab component totally decouples from the "2D" components. In obtaining $\kappa_{\perp}$, the NLGC theory puts no constraints on the turbulence strength in the parallel (slab) direction. The only assumption on the slab direction is that particle motion in this direction can be approximated as a diffusion. This of course remains true when there is enhanced wave intensity. Therefore, the NLGC theory is valid and applicable at an oblique shock. Next we consider which $\lambda_{\|}$should be used in determining $\kappa_{\perp}$. In the NLGC theory, $\lambda_{\|}$enters the nonlinear equation of $\kappa_{\perp}$ through an important approximation of the two-time parallelvelocity autocorrelation function $<v_{z}(t=0) v_{z}\left(t^{\prime}\right)>$ by $\left(v^{2} / 3\right) e^{-v t^{\prime} / \lambda_{\|}}$. This assumes that at large time $t$, the motion of particles in the parallel direction can be regarded as a diffusion process.

Consider now the motion of a particle with energy $E$ upstream the shock, if the wave enhanced turbulent region has a characteristic e-folding distance $d(k)$ (which can be wave number $k$ dependent), then $d(k)$ must satisfy $d(k) \gg \lambda_{\|}^{e}(E)$ for the particle motion to be diffusive in the parallel direction (where the wave number $k$ is related to particle energy $E$ through the resonance condition). If as discussed in the previous sections, the energy break occurs at an energy where the diffusive shock acceleration mechanism breaks down (through e.g., escaping), then replacing $<v_{z}(t=0) v_{z}\left(t^{\prime}\right)>$ by $v^{2} / 3 e^{-v t^{\prime} / \lambda_{\|}^{e}}$ is only valid for $E \ll E_{\text {brk }}$. As $E$ gets closer to $E_{\text {brk }}$, the upstream e-folding distance $d(k)$ becomes closer to $\sim \lambda_{\|}^{e}(E)$, so that the particle motion cannot be fully characterized by a diffusion with $\lambda_{\|}^{e}$. Indeed, when $t \gg d(k) / v$, the particles will spent more time in the less turbulent region in the upstream with a distance $D>d(k)$. At late times, the motion will relax back to the unperturbed diffusion with $\lambda_{\|}^{0}$. Clearly, the problem here is that the enhanced waves only exist a few $\lambda_{\|}^{e}$ in front of the shock, thus replacing $<v_{z}(t=0) v_{z}\left(t^{\prime}\right)>$ by $v^{2} / 3 e^{-v t^{\prime} / \lambda_{\|}^{e}}$ is not always warranted. Since we know at large times $t \rightarrow \infty$, the motion of particles will relax to that governed by the background solar wind turbulence, we can approximate $\lambda_{\|}$by $\lambda_{\|}^{0}$. This approxi- mation represents the upper limit of $\lambda_{\|}$. In contrast, if we use $\lambda_{\|}^{e}$, then it represents the lower limit of $\lambda_{\|}{ }^{7}$

In the following, we consider both choices of $\lambda_{\|}$in calculating the $Q / A$ dependence of the break energy. The difference in these two choices is in $\gamma_{\perp}$ and $\epsilon_{\perp}$ in Equation (23). In the case of $\lambda_{\|}^{0}$ (hereafter case I), we have $\gamma_{\perp}=10 / 9$ and $\epsilon_{\perp}=1 / 9$. In the case $\lambda_{\|}^{e}$ (hereafter case II), we have $\gamma_{\perp}=(5+\beta) / 3$ and $\epsilon_{\perp}=(2+\beta) / 3$.

Figures 1 and 2 plot the $Q / A$ dependence of $E_{0}$ for cases I and II respectively. The $x$-axis is $Q / A$. The $y$-axis is the break energy of ions $E_{0}^{i}$ normalized to that of a proton $E_{0}^{p}$, as calculated from Equation (23). In both the figures, three panels are shown. The left panel has $\beta=-1$, thus $\gamma_{\|}=2, \epsilon_{\|}=1$, corresponding to the Bohm approximation. The middle panel has $\beta=0$, thus $\gamma_{\|}=3, \epsilon_{\|}=2$; the right panel has $\beta=1$, thus $\gamma_{\|}=4$, $\epsilon_{\|}=3$. These represent a broad range of the power-law index $\beta$ of the wave power $I(k) \sim k^{\beta}$. In all the figures, the color of the curves denotes different values of $\xi$. The black curve is for $\xi=0$, corresponding to a parallel shock; the green curve is for $\xi=\infty$, corresponding to a perpendicular shock; and the red and blue curves are for $\xi=1.0$ and $\xi=10$. Also plotted in the figures are the break energies for three events: 2001 April 2, 2002 November 9, and 2002 April 21 respectively. They are represented by the squares, triangles, and stars. In all three events, besides protons and helium, heavy ions $\mathrm{C}, \mathrm{N}, \mathrm{O}$, $\mathrm{Ne}, \mathrm{Mg}, \mathrm{Si}, \mathrm{Ca}$, and $\mathrm{Fe}$ are also included. The charge states are taken from measured $Q / M$ values from SAMPEX for a subset of gradual SEP events where charge measurements are available. A range of $Q / M$ is also shown as the error bar in the $x$-direction (Labrador et al. 2005). The error bars along

\footnotetext{
7 There is no doubt that at lower energies $d(k) \gg \lambda_{\|}^{e}(E)$, where the diffusive shock acceleration is in operation. The transition energy $E$ from using a $\lambda_{\| 1}^{e}(E)$ to a $\lambda_{\| l}^{0}(E)$ is however, hard to estimate without numerically solving the coupled upstream wave action equation and particle transport equation. Note that $\lambda_{\|}^{e}(E)$ is energy dependent and $d(k)$ is $k$ dependent, so $\lambda_{\|}^{e} / d$ can have a complicated energy dependence and may vary from event to event.
} 


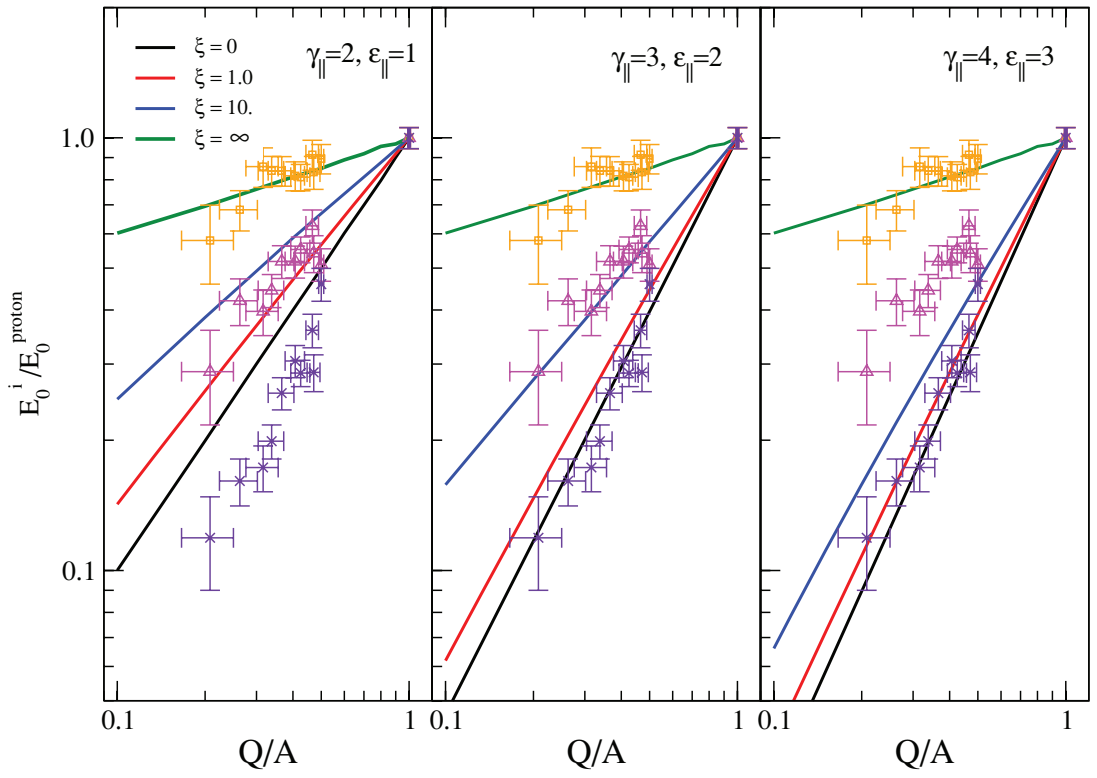

Figure 2. Same as Figure 1, but for the case of $\gamma_{\perp}=(5+\beta) / 3$ and $\epsilon_{\perp}=(2+\beta) / 3$.

Table 1

The Slope of the Break Energy $E_{0}$ as a Function of $Q / A$

\begin{tabular}{lll}
\hline \hline \multicolumn{1}{c}{ Event } & Slope $^{\mathrm{a}}$ & $E_{0}^{\text {proton }}(\mathrm{MeV})$ \\
\hline 2001 Apr 2 & $0.220 \pm 0.056$ & $25.9 \pm 1.3$ \\
2002 Nov 9 & $0.701 \pm 0.060$ & $9.21 \pm 0.47$ \\
2002 Apr 21 & $1.433 \pm 0.060$ & $40.7 \pm 2.1$ \\
\hline
\end{tabular}

Note.

a The slope is obtained as $\frac{d E_{0}}{d(Q / A)}$ for a linear fit following the procedures in Cohen et al. (2005) and Mewaldt et al. (2007).

the $y$-directions represent the uncertainties of the obtained $E_{0}$ using the procedure in Cohen et al. (2005) and Mewaldt et al. (2007). Approximating the observed $E_{0}$ by a power law, using a weighted least-squares fitting (of the $E_{0}$ uncertainties), one can obtain the slopes for the three events, which are listed in Table 1. Comparing to the model calculation, the 2001 April 02 event is likely described by a quasi-perpendicular shock, while the 2002 April 21 event can be described by a quasi-parallel shock with either $\gamma_{\|}=3, \epsilon_{\|}=2$ or $\gamma_{\|}=4, \epsilon_{\|}=3$, but not $\gamma_{\|}=2, \epsilon_{\|}=1$; and the 2002 November 9 event corresponds to an oblique shock. In Tylka et al. (2005), the 2002 April 21 event was interpreted as an "archetypal" quasi-parallel event. Our analysis is consistent with this interpretation. Note from these three events that there does not seem to be a correlation between the proton break energy $E_{0}^{p}$ and the shock geometry. If the break energy can be regarded as a proxy of the event strength, the 2002 April 21 and 2001 April 02 events, which are quasiparallel and quasi-perpendicular according to our analysis, have similar $E_{0}^{p} \mathrm{~s},{ }^{8}$ which are considerably larger than that of the oblique shock case of the 2002 November 9 event.

The differences in Figures 1 and 2 are not significant. This is because the figures are organized by the parameter $\xi=r_{d} \tan ^{2}\left(\theta_{B N}\right)$ (see Equation (24)), which depends on both $r_{d}$ and $\theta_{B N}$. Note by definition that the parallel $(\xi=0)$ and the perpendicular $(\xi=\infty)$ curves in Figures 1 and 2 are identical. The most noticeable difference between Figures 1 and 2 is in the blue curve. It is closer to a straight line in Figure 2 than

8 Indeed, the quasi-parallel 2002 April 21 event has the highest $E_{0}^{p}$. in Figure 1. This is because in the case I, the second term in Equation (23) has a larger contribution than in case II. Indeed, from Equation (23), one can show that as $\xi$ increases, the $Q / A$ dependence of the break energy starts to deviate from linearity. This is clearly seen in Figure 1, where the blue curves are convex.

This convex feature is also seen from observations. Indeed, the 2001 April 2 event (square data points) is a good example. Here the normalized $E_{0}$ s have a weak dependence on $Q / A$ above 0.4 , but slightly increase in the region of $Q / A=0.4$ to 0.3 , followed by a decrease at the two lowest $Q / A$ data points. Ignoring the slight increases of $E_{0}$ between $Q / A=0.4$ to $0.3,{ }^{9}$ the curve is very convex and bounded by the $\xi=10$ (blue) curve and the $\xi=\infty$ (green) curve, suggesting that in this event the main acceleration occurs at a phase when the shock geometry is very perpendicular. Of course, this slight increases of $E_{0}$ between $Q / A=0.4$ to 0.3 could be real. If this is true, this increase cannot be explained by our model, and it suggests that in this event further considerations are needed to understand the underlying acceleration process.

\section{CONCLUSION}

Spectra observed in large SEP events often show spectral breaks which are $Q / A$ dependent. This $Q / A$ dependence of the break (and for that matter, any feature in the spectrum) reflects certain intrinsic properties of the acceleration process. Therefore, careful examination of such $Q / A$ dependences will allow us to better understand the underlying acceleration mechanism. In this paper, we examine how shock geometry may affect the $Q / A$ dependence of the break energy. We find that the $Q / A$ dependence of SEP events varies significantly from a quasi-parallel shock case to a quasi-perpendicular shock case. Using the self-enhanced wave formalism (Lee 1983) for the parallel diffusion coefficient $\kappa_{\|}$and the NLGC theory (Matthaeus et al. 2003; Zank et al. 2004) for the $\kappa_{\perp}$, we explored the $Q / A$ dependence of the energy break for an oblique shock. For a range of $\kappa_{\|}$and $\kappa_{\perp}$, we plot the $Q / A$ dependence of the break energy in Figures 1 and 2. Our results provide a

\footnotetext{
9 This slight increase of $E_{0}$ at the intermediate $Q / A$ range could be due to observation errors, e.g., the measurement of the charge states.
} 
working basis for interpreting the $Q / A$ dependence of the break energy in large SEP events. By comparing our calculations with observations, one can infer some useful information about the geometry of the shock when the acceleration occurs close to the Sun.

This work is supported in part by NASA grants NNX06AC21G, NNH07ZDA001N-HGI and NNX07AL52A for G.L., O.V., and G.P.Z. at UAH; NNX06AC21G, NNX8AI11G, and NNX07AP69G for RAM and CMSC at Caltech; Caltech subcontract 44A-1081056 for G.M.M. at A.P.L., and NNH07ZDA001N-HGI for M.I.D. at SWRI. The authors acknowledge discussions with M.A. Lee and an anonymous referee for valuable comments. G.L. thanks Dr. Yan Wang for some very stimulating discussions.

\section{REFERENCES}

Axford, W. I., Leer, E., \& Skadron, G. 1977, Proc. 15th Int. Cosmic Ray Conf (Plovdiv), 11, 132

Band, D., et al. 1993, ApJ, 413, 281

Bell, A. R. 1978a, MNRAS, 182, 147

Bell, A. R. 1978b, MNRAS, 182, 443

Bieber, J., et al. 1994, ApJ, 420, 294

Blandford, R. D., \& Ostriker, J. P. 1978, ApJ, 221, L29

Channok, C., Ruffolo, D., Desai, M. I., \& Mason, G. M. 2005, ApJ, 633, L53

Cohen, C., Cohen, C. M. S., Mewaldt, R. A., Cummings, A. C., Leske, R. A., Stone, E. C., von Rosenvinge, T. T., \& Wiedenbeck, M. E. 2003, Adv. Space Res., 32, 2649

Cohen, C., et al. 2005, J. Geophys. Res., 110, A09S16

Drury, L.O'C. 1983, Rep. Prog. Phys., 46, 973

Ellision, D. C., \& Ramaty, R. 1985, ApJ, 298, 400

Forman, M., \& Webb, G. 1985, in Collisionless Shocks in the Heliosphere: A Tutorial Review Geophysical Monograph, Vol. 34, ed. R. G. Stone \& B. T. Tsurutani (Washington, DC: American Geophysical Union), 91

Forman, M., Webb, G., \& Axford, W. I. 1981, Proc. 17th Int. Cosmic Ray Conf. (Paris), 9, 238

Gordon, B. E., Lee, M. A., E. Mobius, \& Trattner, K. J. 1999, J. Geophys. Res., 104,28263
Ip, W.-I., \& Axford, W. I. 1992, AIP Conf. Proc. 264, Particle Acceleration in Cosmic Plasmas, ed. G. P. Zank \& T. K. Gaisser (Melville, NY: AIP), 400

Krymsky, G. F. 1977, Doklady Akad. Nauk. SSSR, 234, 1306

Lee, M. A. 1983, J. Geophys. Res., 88, 6109

Labrador, A. W., Leske, R. A., Mewaldt, R. A., Stone, E. C., \& von Rosenvinge, T. T. 2005, Proc. 29th Int. Cosmic Ray Conf., (Pune), 1, 99

Li, G., Hu, Q., \& Zank, G. P. 2005a, in AIP Conf. Proc. 781, The Physics of Collisionless Shocks, ed. G. Li, G. P. Zank, \& C. T. Russell (Melville, NY: AIP), 233

Li, G., Zank, G. P., \& Rice, W. K. M. 2003, J. Geophys. Res., 108, 1082

Li, G., Zank, G. P., \& Rice, W. K. M. 2005b, J. Geophys. Res. A, 110, 06104

Lee, M. 1983, J. Geophys. Res., 88, 6109

Lee, M. 2005, ApJS, 158, 38

Manchester et al. 2005, ApJ, 622, 1225

Mason, G. M., Desai, M. I., Cohen, C. M. S., Mewaldt, R. A., Stone, E. C., \& Dwyer, J. R. 2006, ApJ, 647, L65

Matthaeus, W., Qin, G., Bieber, J. W., \& Zank, G. P. 2003, ApJ, 590, L53

Mazur, J. E., Mason, G. M., Klecker, B., \& McGuire, R. E. 1992, ApJ, 401, 398

McKenzie, J. F., \& Westphal, K. O. 1969, Planet. Space Sci., 17, 1029

Mewaldt, R. A., Cohen, C. M. S., Haggerty, D. K., Li, G., \& Mason, G. M. 2007, in Proc. 30th Int. Cosmic Ray Conf. 1, 99

Mewaldt, R. A., et al. 2005a, J. Geophys. Res. A, 110, 09S18

Mewaldt, R. A., et al. 2005b, in AIP Conf. Proc. 781, The Physics of Collisionless Shocks, ed. G. Li, G. P. Zank, \& C. T. Russell (New York: AIP), 227

Ng, C. K., \& Reames, D. V. 2008, ApJ, 686, L123

Ng, C. K., Reames, D. V., \& Tylka, A. J. 2003, ApJ, 591, 461

Rice, W. K. M., Zank, G. P., \& Li, G. 2003, J. Geophys. Res., 108, 1369

Schachi, A. 2006, A\&A, 453, L43

Schachi, A., Bieber, J., Matthaeus, W. H., \& Qin, G. 2004, ApJ, 616, 617

Tylka, A. J., Boberg, P. R., McGuire, R. E., Ng, C. K., \& Reames, D. V. 2000, in AIP Conf. Proc. 528, Acceleration and Transport of Energetic Particles Observed in the Heliosphere, ed. R. A. Mewaldt et al. (Melville, NY: AIP), 147

Tylka, A. J., Cohen, C. M. S., Dietrich, W. F., Lee, M. A., Maclennan, C. G., Mewaldt, R. A., Ng, C. K., \& Reames, D. V. 2005, ApJ, 625, 474

Tylka, A. J., Cohen, C. M. S., Dietrich, W. F., Lee, M. A., Maclennan, C. G., Mewaldt, R. A., Ng, C. K., \& Reames, D. V. 2006, ApJS, 164, 536

Tylka, A. J., \& Lee, M. A. 2006, ApJ, 646, 1319

Zank, G. P., Rice, W. K. M., \& Wu, C. C. 2000, J. Geophys. Res., 101, 17093

Zank, G. P., et al. 2004, J. Geophys. Res., 101, 17093 